\title{
L-proline and betaine inhibit extracellular enzymes mediated abeta 1-42 aggregation, oxidative stress, and toxicity
}

\section{Tania Ismail | Sushma Reddy Vancha | Mathumai Kanapathipillai ${ }^{\circledR}$}

University of Michigan-Dearborn, Department of Mechanical Engineering, Dearborn, Michigan

\section{Correspondence}

Mathumai Kanapathipillai, University of Michigan-Dearborn, Department of

Mechanical Engineering, Dearborn, MI, 48128.

Email: mathumai@umich.edu

Funding information

University of Michigan-Dearborn, Grant/ Award Number: Start-up fund; College of Engineering and Computer Science; department of Mechanical engineering

\begin{abstract}
Extracellular amyloid plaques are characteristics of Alzheimer's disease (AD). The amyloid plaques mainly consist of amyloid- $\beta$ (abeta) fragments. Extracellular biomolecules play a key role in abeta aggregation and subsequent plaque formation during AD. Among them, extracellular enzymes are known to induce abeta aggregation by catalyzing the crosslinking of abeta proteins. Although several therapeutic strategies have been studied to combat $A D$, studies to treat extracellular enzymes mediated abeta aggregation and toxicity is not explored in detail yet. Here we study the potential of osmolyte molecules I-proline and betaine to inhibit the abeta aggregation and toxicity promoted by extracellular enzymes transglutaminase and lysyl oxidase in vitro. Results reveal that I-proline and betaine have the potential to inhibit extracellular matrix enzymes mediated abeta aggregation/fibril formation and toxicity and oxidative stress in brain endothelial cells. Hence osmolytes could have the potential to modulate the effects of extracellular molecules during $A D$.
\end{abstract}

\section{KEYWORDS}

I-proline, abeta peptide, aggregation, alzheimer's, betaine, extracellular enzymes

\section{1 | INTRODUCTION}

Alzheimer's disease is a neurological disorder that affects around $10 \%$ of the population of age over 65 , that costs billions of dollars worldwide. The disease is characterized by senile plaques and neurofibrillary tangles in the brain tissue. ${ }^{[1-4]}$ The plaques are predominantly composed of the amyloid plaques containing 39-42 residues peptide termed beta-amyloid peptide (abeta), produced by the proteolysis of beta-amyloid precursor protein in soluble form. ${ }^{[5,6]}$ Among the several abeta fragments generated during this proteolysis process, the 42-amino acids version (abeta 42) had shown to be found in high amount in amyloid plaques, and have aggregation tendency. ${ }^{[1,5]}$ The amino acid residues 29-42 consists a cluster of nonpolar amino acids that could play a role in the amyloid formation. ${ }^{[2,7]}$

Several extracellular matrix factors play a role in promoting and stabilizing abeta aggregation. ${ }^{[8-10]}$ Among them extracellular enzymes are one of the key constituents found colocalized with abeta aggregates. $^{[9]}$ Extracellular enzymes transglutaminase (TG), and lysyl oxidase (LOX) are known to crosslink the extracellular matrix and modulate the extracellular matrix (ECM) during diseases. ${ }^{[9,11,12]}$ Recent studies report that both TG, and LOX have been observed in Alzheimer's disease brain tissues. ${ }^{[11,13-15]}$ Transglutaminase is believed to contribute to Alzheimer's pathology by either directly binding to abeta, or modulating the abeta cascade and neurotoxicity by crosslinking the ECM. ${ }^{[16]}$ The result is the production of neurotoxic and protease resistant abeta. Lysyl oxidase, which is known to play a major role in collagen crosslinking also believe to contribute to abeta pathology in a similar manner. ${ }^{[11]}$

Here, in this article, we study the effect of extracellular enzymes transglutaminase and lysyloxidase on the aggregation of abeta (1-42) peptide. In addition, we study the potential of natural osmolytes betaine and I-proline in inhibiting transglutaminase and lysyl oxidase mediated aggregation and fibrillization. Proline and betaine are known to influence protein solvation, prevent aggregation, and misfolding. ${ }^{[17]}$ Further, recently it has been shown they have inhibitory potential on human serum albumin, and insulin aggregation/ 
fibril formation. ${ }^{[18,19]}$ Hence, they could have inhibitory effect on extracellular enzyme mediated abeta aggregation. The studies were carried out using thioflavin T (ThT), dynamic light scattering (DLS), atomic force microscopy (AFM), transmission electron microscopy (TEM), cell toxicity assay (XTT), and oxidative stress assays. The results show that transglutaminase (TG), and LOX promote the aggregation and fibrillization of abeta peptide, oxidative stress, and toxicity. Further, betaine (B) and I-proline (LP) inhibited the effect of TG and LOX induced abeta peptide aggregation/fibrillization, oxidative stress, and toxicity.

\section{2 | MATERIALS AND METHODS}

\section{1 | Materials}

Abeta peptide (1-42), ultra pure (1,1,1,3,3,3-Hexafluoro-2-propanol [HFIP] treated), is purchased from rPeptide. Transglutaminase (TG) was purchased from either modernized pantry LLC or Ajinomoto. Human Lysyl oxidase peptide (LOX- [EDTSCDYGYHRRFA]) was custom synthesized from Genscript. L-proline (LP) and betaine (B), and all other chemicals were purchased from Sigma Aldrich (St. Louis, MO).

\section{2 | Stock solutions preparation}

A $1 \mathrm{mg}$ of abeta peptide (1-42) was dissolved in $500 \mu \mathrm{L}$ HFIP. Then, the solution was aliquoted into 10 vials $(100 \mu \mathrm{g} / \mathrm{vial})$, and was kept in a chemical hood for complete evaporation of HFIP overnight. One vial of abeta peptide was dissolved in $500 \mu \mathrm{L} \mathrm{HCl}(10 \mathrm{mM})$ and $10 \mu \mathrm{L}$ of DMSO to get a final concentration of $48 \mu \mathrm{M}$ (stock). For transglutaminase (TG) $5 \%$ of solution was freshly prepared by dissolving $50 \mathrm{mg} /$ $\mathrm{mL}$ in water $(\mathrm{pH} 7)$, and LOX stock solution $(10 \mathrm{mM})$ was prepared by dissolving in water $(\mathrm{pH}$ 7). Betaine (B) and L-proline (LP) were either dissolved in water $(\mathrm{pH} 7)$ or dissolved in $\mathrm{HCl}(10 \mathrm{mM})$ and DMSO to a final concentration of $10 \mathrm{M}$.

\section{3 | Thioflavin-T (ThT) fluorescence measurement}

For the ThT measurement aggregation study, $50 \mu \mathrm{L}$ of $48 \mu \mathrm{M}$ abeta peptide (1-42) was dissolved in $10 \mathrm{mM} \mathrm{HCl}$ and $2 \%$ DMSO, with and without TG or LOX at $(0.025,0.05,0.01,0.1 \mathrm{w} / \mathrm{v} \%)$, and B or LP of $100 \mathrm{mM}$ final concentrations to make the final working solution. The samples were kept to aggregate for 24 hours in an incubator at $37^{\circ} \mathrm{C}$. Next, $100 \mu \mathrm{M}$ concentration of ThT solution was prepared in $50 \mathrm{mM}$ Tris buffer, $\mathrm{pH}$. The aggregated abeta samples $(5 \mu \mathrm{L})$ were then mixed with $90 \mu \mathrm{L}$ of the ThT solution, and the ThT fluorescence was measured using a spectramax M3 spectrophotometer at 440/482 nm excitation and emission. Aggregation end points were measured at 24 and 48 hours. Three independent experiments were done and the average readings were obtained.

\subsection{AFM}

AFM images were obtained using Hitachi AFM5100N in the lab. Abeta peptide $(48 \mu \mathrm{M})$ was aggregated in the presence of $0.1 \% \mathrm{TG}$ or LOX, and with and without the small molecule drugs betaine and I-proline for 24 hours. Then, $5 \mu \mathrm{L}$ of the solutions were spotted on freshly cleaved mica and kept to dry before imaging. Tapping mode was used to image the samples, and AFM tips (Nanosensor, CA), with a resonance frequency of $45-115 \mathrm{kHz}$ was used. The average length and width of the cantilever is 225 and $28 \mu \mathrm{m}$, and the force constant of the tip is $0.5-9.5 \mathrm{~N} \mathrm{~m}^{-1}$. A scan area of $1 \times 1$ or $5 \times 5 \mu \mathrm{m}$ is imaged for at least two different samples of each condition.

\section{5 | TEM}

Peptide samples were aggregated in the presence of TG or LOX, and with and without osmolytes betaine and I-proline for 24 hours as described in the AFM sample preparation. Then, $5 \mu \mathrm{L}$ of the solutions were spotted on holy carbon grids and stained with phosphotungstic acid. TEM images were obtained using JEOL JEM 1400 microscope in the MIL facility at UM-Ann Arbor, MI.

\subsection{DLS}

For the DLS measurements, samples were prepared under similar conditions used for the AFM and TEM study. The measurements were carried out in a Malvern zetasizer instrument in the lab. At least three independent experiment samples were measured and the zeta average size measurements were obtained.

\section{7 | DCFH-DA assay}

The effect of oxidative stress of abeta peptide, in the presence of extracellular enzymes, and small molecule drugs on human brain microvascular endothelial cells (hBMVEC) was studied by DCFH-DA assay. Quantifying oxidative stress by 2,7-dichlorodihydrofluorescein (DCFH)-based fluorescent probes has been widely reported. ${ }^{[20,21]}$ For the study, hBMVEC cells were used. The cells were kindly provided by Dr. Kalyan Kondapalli at UM-Dearborn, and were cultured in medium containing $\mathrm{M}-199$, supplemented with $10 \% \mathrm{FBS}$, and $5 \%$ PenStrep according to the standard protocol. For this experiment, $2 \times 10^{4}$ cells/well were cultured in a 96 well plate for 24 hours overnight. Cells were then treated with final concentrations of abeta peptide $(2 \mu \mathrm{M})$, TG or LOX $(0.005 \%)$, and the small molecule osmolyte drugs ( $5 \mathrm{mM}$ ), and subsequently were incubated for 48 hours. DCFHDA assay fluorescence was performed at $485 / 535 \mathrm{~nm}$, excitation and emission. Three independent DCFH-DA experiments were performed and the average readings were obtained.

\section{8 | CellROX}

As a complementary method to DCFH-DA assay, CellROX experiment, which was widely used assay to determine oxidative stress, was performed as per manufacturer's (Thermofisher scientific, Waltham, MA) protocol. Briefly, human brain microvascular endothelial cells (hBMVEC) were cultured in eight well at $1 \times 10^{4}$ cells $/$ well. Then, after 24 hours, abeta, abeta+LOX, abeta+ TG, with and without betaine, and I-proline were added to the cells at similar concentrations reported in the DCFH-DA assay method. After 48 hours of treatment, 
CellROX green was added at $5 \mu \mathrm{M}$ concentration for 30 minutes. Cells were then fixed with formaldehyde and imaged using Nikon A1 confocal microscope.

\section{9 | Immunofluorescence}

hBMVEC cells were cultured in eight well chambers at $2 \times 10^{4}$ cell density, and abeta with and without extracellular enzymes, and osmolytes were added to the wells. Cells were then incubated for 48 hours. Immunostaining of ZO-1 (purchased from cell signaling technology) was performed following standard staining protocol. Immunofluorescence images of tight junctions were obtained using a Nikon A-1 spectral confocal microscope at the UM-Ann Arbor microscopy image analysis laboratory (MIL), and images were obtained for two different samples of each condition.

\subsection{0 | Cell toxicity assay}

The toxicity of $A \beta$ (1-42) fibrils in the presence of extracellular enzymes and osmolytes on human brain microvascular endothelial cells (hBMVEC) was studied by alamar blue assay. Cells were cultured at $2 \times 10^{4}$ cells/well in a 96 well plate overnight. For the toxicity study, abeta with and without extracellular enzymes and small molecule drugs were prepared in $100 \mathrm{mM} \mathrm{NaCl}$ and $100 \mathrm{mM}$ of $\mathrm{NaHPO} 4$ buffer, at $\mathrm{pH}$ 7. Because of the high concentrations tested in the cells, abeta was dissolved in buffer solution instead of DMSO and $\mathrm{HCl}$ mixture used for the ThT and oxidative stress experiments. Cells were treated with, abeta peptide concentrations $(2.5,5,10$, and $12.5 \mu \mathrm{M})$, with and without, TG (0.02\%), LOX (0.02\%) and small molecule drugs $(2 \mathrm{mM})$, and subsequently were incubated for 48 hours. The Alamar blue assay was analyzed by the fluorescent dye indicator resazurin at 570/590 nm excitation/emission according to the manufacturer's protocol using a M3 spectramax spectrophotometer.

\subsection{1 | Statistical analysis}

In this study, each experiment was conducted at least 3 times, and they are presented as mean \pm SE of the mean (SEM). $P$ values were determined from the results of at least three independent experiments. Statistical significance was computed using either by student $t$ test or by analysis of variance (ANOVA) followed by Tukey's HSD post hoc analysis test. $* * P<.01$, and $* P<.05$ are considered as significance.

\section{3 | RESULTS AND DISCUSSION}

Here in this study, we tested the influence of extracellular enzymes, and osmolyte molecules on abeta aggregation, fibril formation, and oxidative stress. The aggregation studies were analyzed by thioflavin T, AFM, TEM, and DLS measurements. First, we tested whether TG and LOX promote aggregation of the abeta peptides by thioflavin $\mathrm{T}$ assay. For the thioflavin T study, $45 \mu \mathrm{M}$ of abeta peptide samples aggregated in the presence of three different concentrations of TG and LOX to see the inhibitory effects. The thioflavin T measurements of abeta aggregation show that the both TG and LOX significantly promote abeta aggregation at $0.1 \% \mathrm{w} \mathrm{v}^{-1}$ concentrations (Figure 1A,B). Based on the ThT fluorescence, we chose $0.1 \%$ of TG and LOX concentrations for the subsequent inhibition studies with the osmolyte molecules. Both I-proline and betaine at $100 \mathrm{mM}$ of concentrations inhibits the extracellular enzymes mediated abeta aggregation (Figure $2 A, B$ ).

The findings were further corroborated with AFM and TEM images and DLS measurements. From Figure 3 it can be seen from the AFM images that TG and LOX promote fibrillar morphology of abeta. When aggregated in the presence of I-proline or betaine less fibril or aggregation was observed (Figure 3). Similar observations were observed from TEM images (Figure 4). As can be seen, abeta peptides when treated with TG and LOX showed more aggregates/fibrillar structures, whereas in the presence of TG or LOX, very few aggregates/fibrils were observed. DLS measurements were also in agreement with the ThT, AFM, and TEM observations. Figure 5A,B reveals an increase in size of the abeta peptide aggregates when treated with LOX or TG. The average aggregate sizes were reduced in the presence of osmolytes. The aggregations studies indicate that the extracellular enzymes do play a role in abeta aggregation, and the effect could be significantly reduced in the presence of osmolytes betaine and Iproline.

Studies report that abeta aggregates causes oxidative stress by interacting with the cell lipid membrane, leading to free radical chain reactions and ultimately oxidative stress. ${ }^{[22,23]}$ To determine whether abeta, with and without the extracellular enzymes and small molecules exhibit oxidative stress, next we performed oxidative stress measurements with DCFH-DA and CellROX assays. For the study, human brain microvascular endothelial cells (hBMVEC) were used. First, we tested the effect of TG and LOX induced oxidative stress by DCFHDA assay. As can be seen from Figure 6A, transglutaminase indeed promote oxidative stress of abeta aggregates. The oxidative stress effects were significantly inhibited in the presence of I-proline and betaine (Figure 6A). Similar observations were found with lysyl oxidase treated samples. Cells treated with abeta and lysyl oxidase exhibited enhanced oxidative stress. When treated with the osmolytes significant inhibition in the oxidative stress was observed (Figure 6B). Next, CellROX green staining experiments were performed to further confirm the findings. As depicted in Figure 7, TG and LOX significantly increase the oxidative stress evidenced by the increase in fluorescence. Further, in agreement with the DCFH-DA assay, the oxidative stress seems to be inhibited with betaine and I-proline as evidenced by the low CellROX signal. Betaine and I-proline are known to reduce oxidative stress. ${ }^{[2,25]}$ And from this study, it is also revealed that they also have the ability to reduce the oxidative stress in the presence of extracellular enzymes.

Abeta aggregation is also known to induce endothelial dysfunction, and disruption of blood brain barrier. ${ }^{[26,27]}$ To assess whether abeta and extracellular enzymes play a role in blood brain barrier damage, we performed immunofluorescence staining of ZO-1. The cytoplasmic accessory protein ZO-1, under normal conditions distribute continuously around the cell membrane. ${ }^{[28]}$ However, in the presence of abeta, it has been shown that the tight junctions were disrupted, and broken ZO-1 links were observed. ${ }^{[28]}$ Similarly, in this study as 


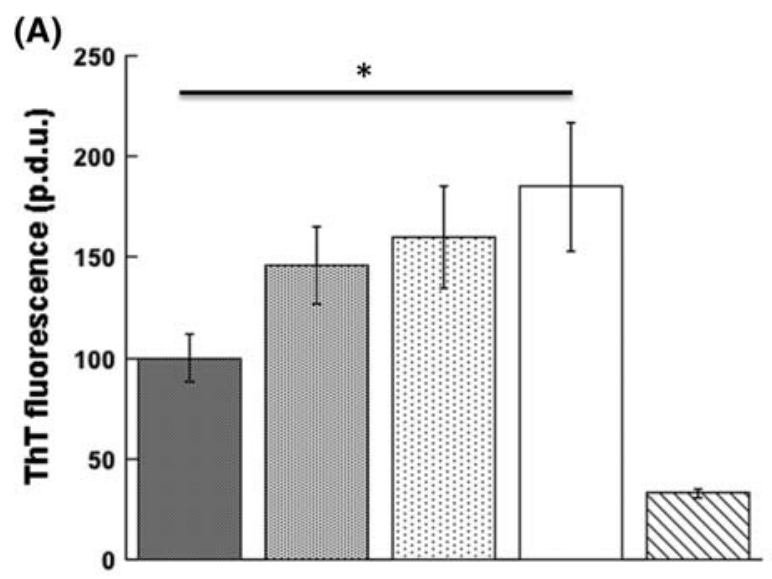

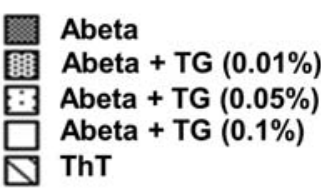
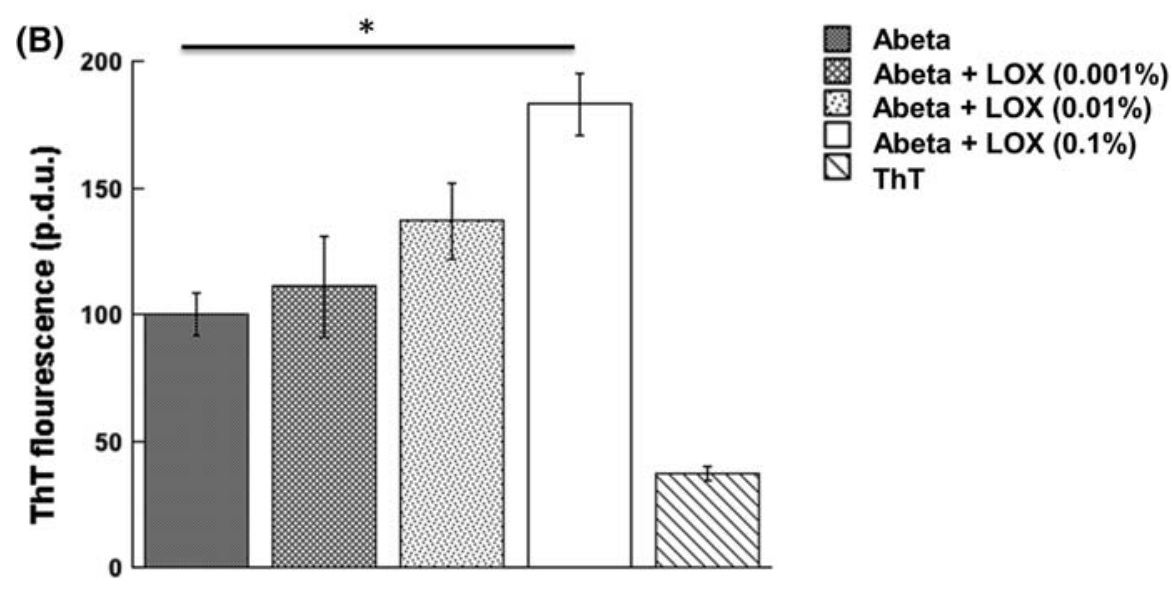

FIGURE 1 Thioflavin-T fluorescence measurements of abeta peptide aggregation with and without extracellular enzymes. (A) Abeta peptide $(45 \mu \mathrm{M})$ aggregation in the presence of $0.1 \%, 0.05 \%$, and $0.01 \%$ transglutaminase (TG) concentrations. (B) Abeta peptide (45 $\mu \mathrm{M})$ aggregation, in the presence of $0.001 \%, 0.01 \%$, and $0.1 \%$ of lysyl oxidase (LOX) concentrations. ThT fluorescence measurements indicate that the abeta aggregation is promoted in the presence of extracellular enzymes. Data with significance compared to abeta are represented as ${ }^{*} P<.05$

can be seen from Figure 6, when the cells were treated with abeta alone or abeta with TG/LOX, the ZO-1 staining is diffused compared to the control cells without any treatment. Moreover, when the cells were co-treated with osmolytes betaine of I-proline, significant restoration of cell junctions were observed (Figure 8). The results indicate that the osmolytes could have the capability to mitigate the blood brain barrier damage because of abeta aggregation. This finding is in corroboration with that of oxidative stress findings, where increase in the oxidative stress was observed in the presence of extracellular enzymes, while it was significantly inhibited when treated with small molecule drugs.

Finally, we tested the toxicity effects of abeta with and without extracellular enzymes and small osmolyte molecules. One of the major effects of abeta aggregation is cellular toxicity and death, ${ }^{[29,30]}$ hence it is important to study the abeta aggregation effects on cell viability. For the toxicity study, we used human brain microvascular endothelial cells (hBMVEC). Cells were treated with abeta and combination of the enzymes and drugs for 48 hours, and XTT assay was performed. As can be seen from Figure 9, at higher concentrations of abeta significant toxicity was observed (Figure 9A). The toxicity was further increased in the presence of TG or LOX, and with the treatment of osmolytes drug molecules, it was reduced significantly. The study shows that the abeta toxicity is, influenced by the extracellular enzymes. Further, the results show the potential of osmolytes betaine and I-proline in inhibiting the endothelial cell toxicity induced by abeta aggregation due to TG and LOX.

In this article, we have shown that extracellular enzymes transglutaminase and lysysl oxidase have the potential to promote aggregation of abeta peptide, which in turn contributes to oxidative stress and toxicity in normal brain endothelial cells. TG and LOX are known to crosslink extracellular proteins and have been known to contribute to diseases by modulating the extracellular matrix properties. ${ }^{[31]}$ Further, recent findings show that TG and LOX enzymes have been found colocalized in Alzheimer's disease patients. ${ }^{[11,32]}$ They may either have a direct or indirect effect, or both on abeta aggregation and subsequent amyloid formation, leading to AD. In the case of a direct effect, they may play a role either in the abeta-abeta association/nucleation via acting as a template, or by crosslinking the abeta proteins similar to their extracellular proteins crosslinking mechanisms. On the other hand if it is an indirect effect, by crosslinking and forming a highly dense ECM matrix, they may promote abeta accumulation in the ECM parenchyma, thereby preventing abeta clearance. This will in turn will lead to, abeta nucleation, aggregation, and subsequent amyloid plaque 

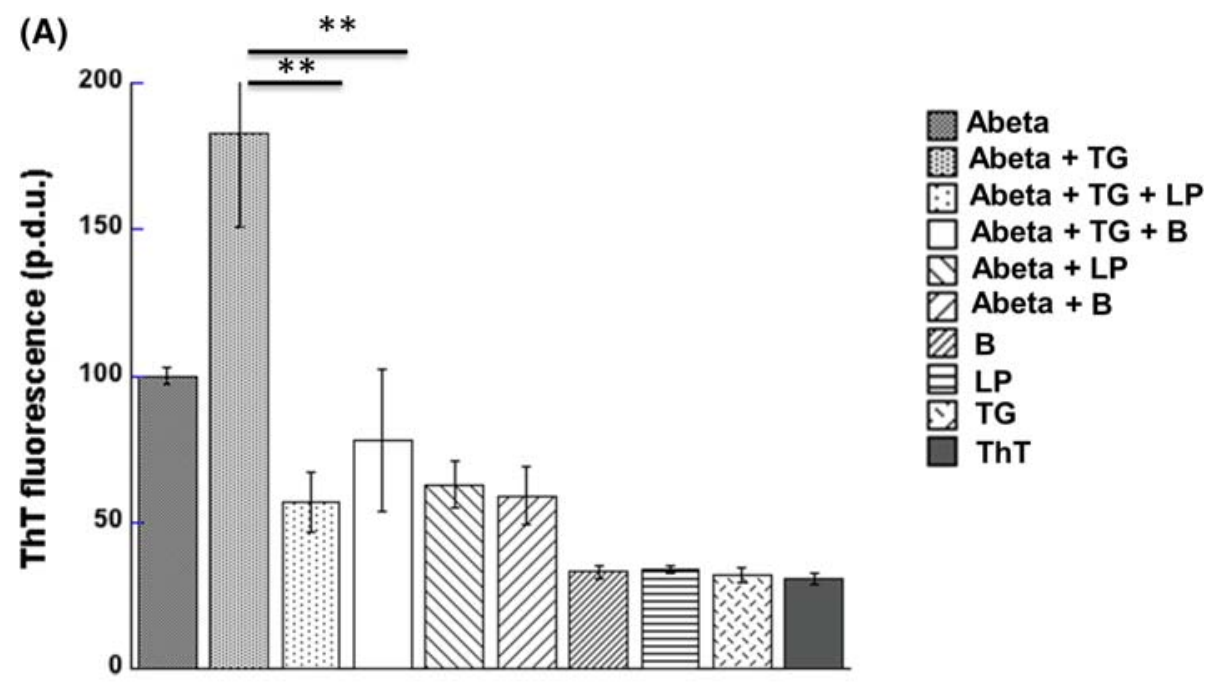

(B)

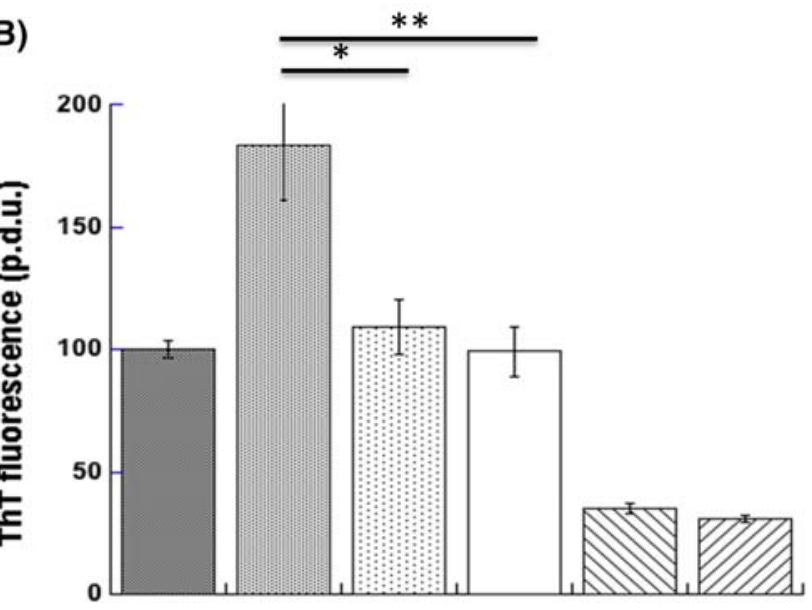

FIGURE 2 Thioflavin-T fluorescence measurements of abeta peptide aggregation with and without extracellular enzymes and osmolytes. (A) Abeta peptide $(45 \mu \mathrm{M})$ aggregation in the presence of $0.1 \%$ transglutaminase and $100 \mathrm{mM}$ of osmolytes I-proline and betaine. (B) Abeta peptide $(45 \mu \mathrm{M})$ aggregation in the presence of $0.1 \%$ lysyl oxidase, and $100 \mathrm{mM}$ of osmolytes I-proline or betaine. ThT fluorescence measurements indicate that the abeta aggregation is modulated by the presence of extracellular enzymes and osmolytes. Data with significance are represented as ${ }^{* *} P<.01$ and ${ }^{*} P<.05$

(A) Abeta

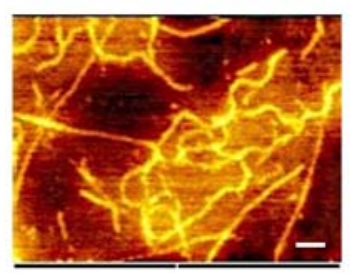

(B)

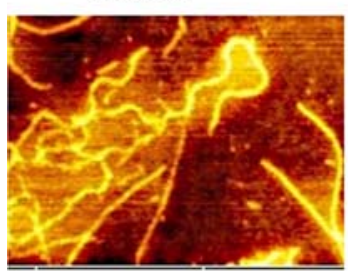

Abeta + TG

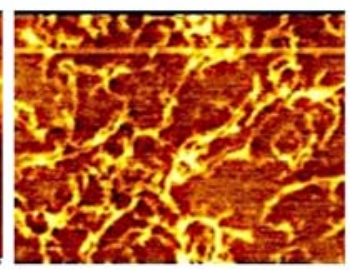

Abeta + LOX

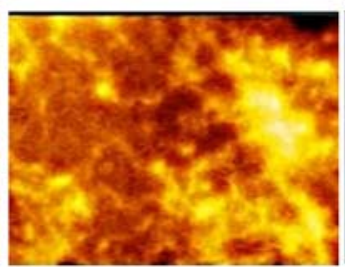

Abeta +TG +LP

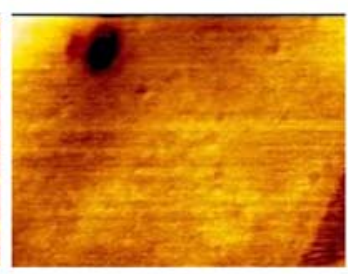

Abeta +TG +B

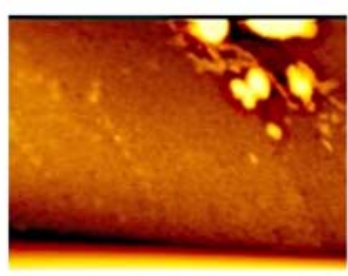

Abeta + LOX + B

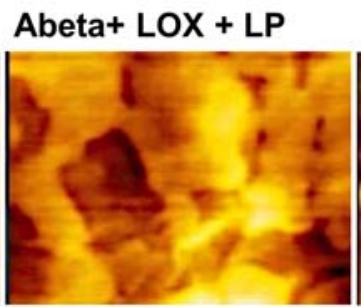

Abeta

Abeta + LOX

+ LOX + LP

ThT

FIGURE 3 Morphology of abeta peptide $(45 \mu \mathrm{M})$ aggregation in the presence of TG, LOX, and osmolytes I-proline and betaine, imaged by atomic force microscopy. The images reveal abeta aggregation/fibrillization is promoted in the presence of TG and LOX, and inhibited in the presence of I-proline. Scale bar $100 \mathrm{~nm}$ 
(A) Abeta

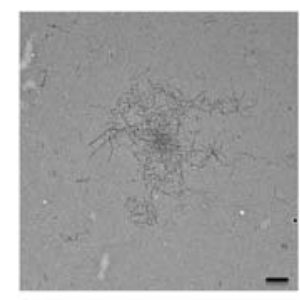

(B) Abeta

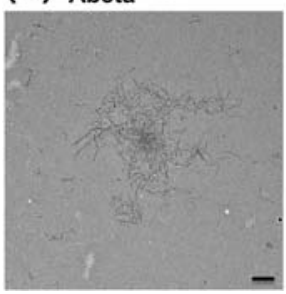

Abeta + TG

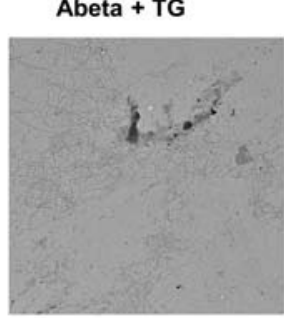

Abeta + LOX

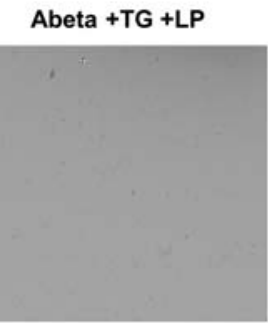

Abeta + LOX + LP
Abeta +TG +B

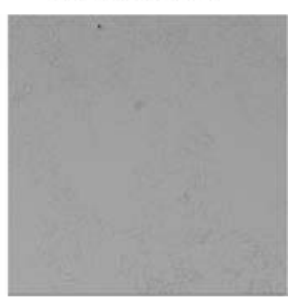

Abeta + LOX + B
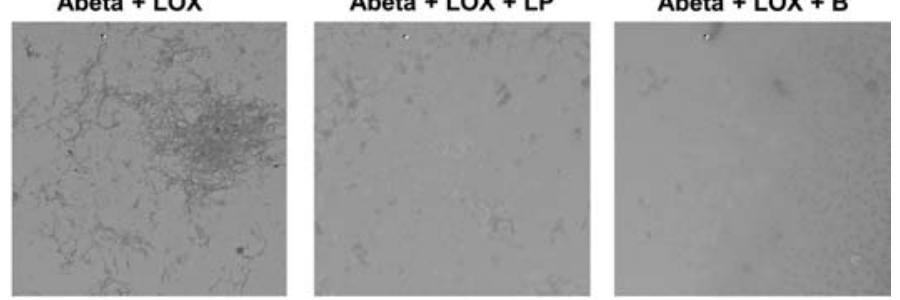

FIGURE 4 Transmission electron microscopy images of abeta peptide $(45 \mu \mathrm{M})$ aggregation in the presence of TG, LOX, and osmolytes LP and B. The images reveal abeta aggregation/fibrillization is promoted in the presence of TG and LOX, and inhibited in the presence of I-proline, and betaine, in agreement with AFM images. Scale bar $100 \mathrm{~nm}$

formation. From our in vitro experiments, the results suggest the enzymes could have a direct influence on abeta aggregation. However, to understand the complete mechanism, detail studies have to be performed both in vitro, and in cellular environment under relevant physiological conditions mimicking the normal and Alzheimer's disease states.

Further, our studies show osmolytes I-proline and betaine significantly inhibited the extracellular enzymes mediated aggregation. Osmolytes are known to protect protein from stressful conditions, and has been shown that several osmolytes have the ability to prevent or inhibit protein aggregation. ${ }^{[3,34]}$ The studies report that osmolytes modulate the solvation state of the amyloidogenic protein or peptides thereby affecting the aggregation pathway. The small molecules proline and betaine investigated here, known to have chaperone like properties, as well as antioxidative properties. ${ }^{[34,35]}$ Proline has been shown to protect oxidative stress by scavenging reactive oxygen species by forming stable free radicals adducts of proline. ${ }^{[25]}$ A recent study on betaine, when cotreated with Rotenone (a pesticide known to induce reactive oxygen species), revealed neuroprotective and antioxidant properties in PC12 cells, by inhibiting mitochondrial superoxide generation. ${ }^{[36]}$ In the current study, both proline and betaine seems to exhibit both chaperone and antioxidant-like properties as seen from significant inhibition in aggregation, and oxidative stress when abeta is aggregated in the presence of these osmolytes.

\section{4 | CONCLUSIONS}

Here, we show that extracellular enzymes have the capability to influence the abeta peptides aggregation, fibrillization, and oxidative stress in vitro. Extracellular enzymes have been shown to colocalize with amyloid plaques, and subsequently crosslink them and prevent them from degradation. This in turn leads to excessive oxidative stress and neuronal death. This article's study shows that in the presence of osmolytes, the extracellular enzymes mediated

(A)
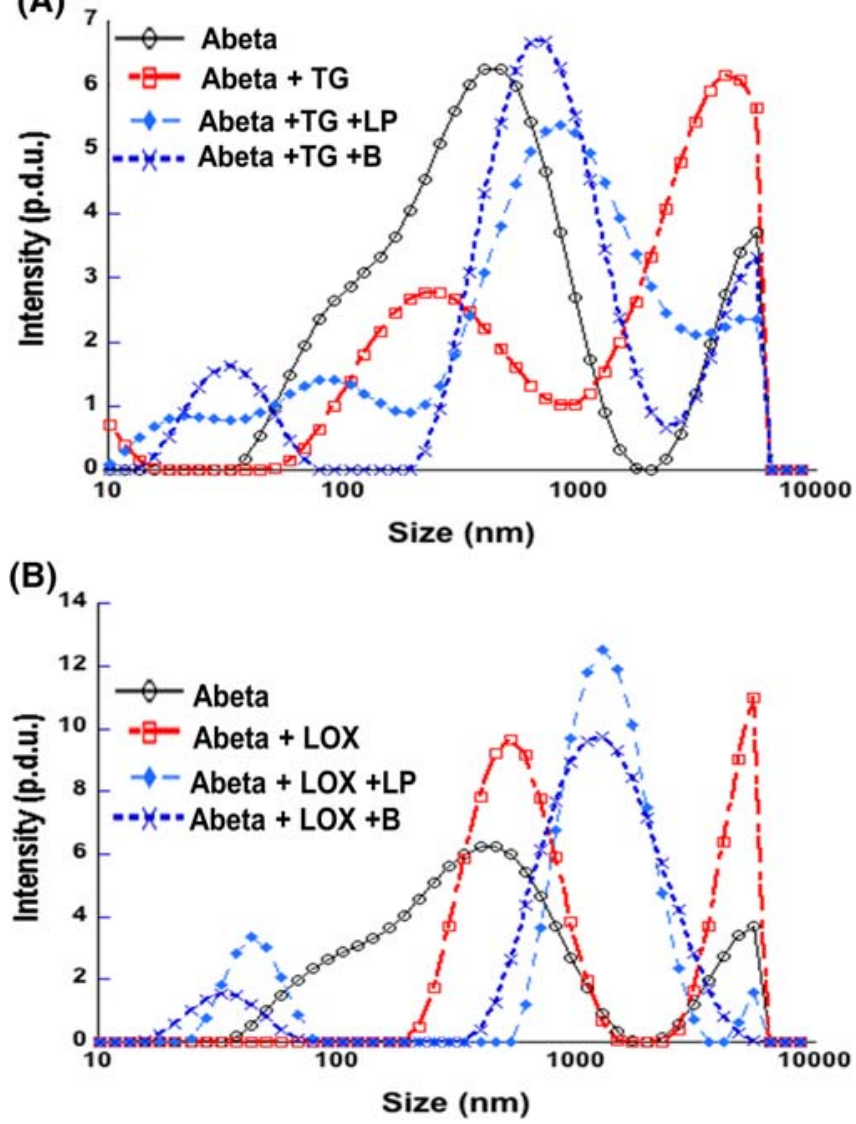

FIGURE 5 DLS measurements abeta peptide $(45 \mu \mathrm{M})$ aggregation in the presence of TG, LOX, and osmolytes LP and B. The size measurements show size increase of abeta aggregation/fibrillization in the presence of TG and LOX. Decrease in the size of the aggregates was observed in the presence of I-proline and betaine 


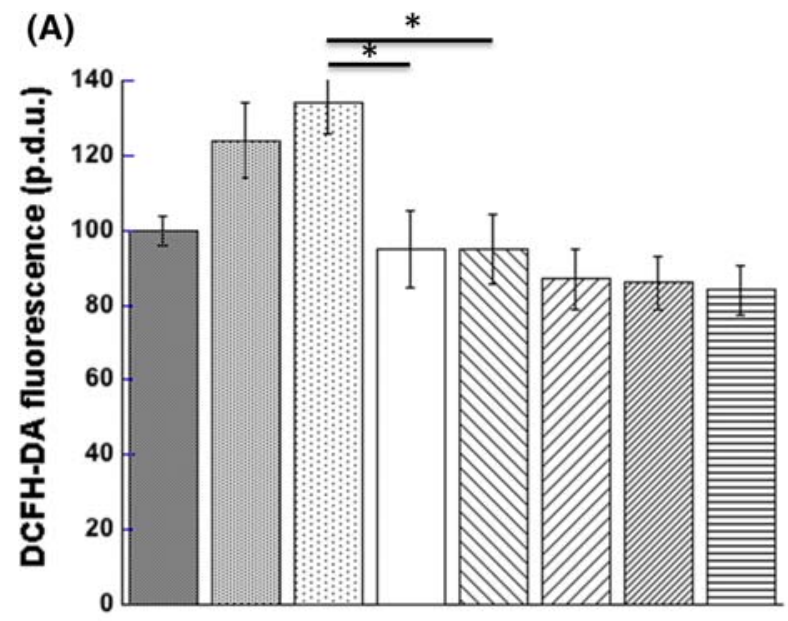

No treatment
Abeta
Abeta + TG
Abeta + TG + LP
Abeta + TG + B
TG
B
LP
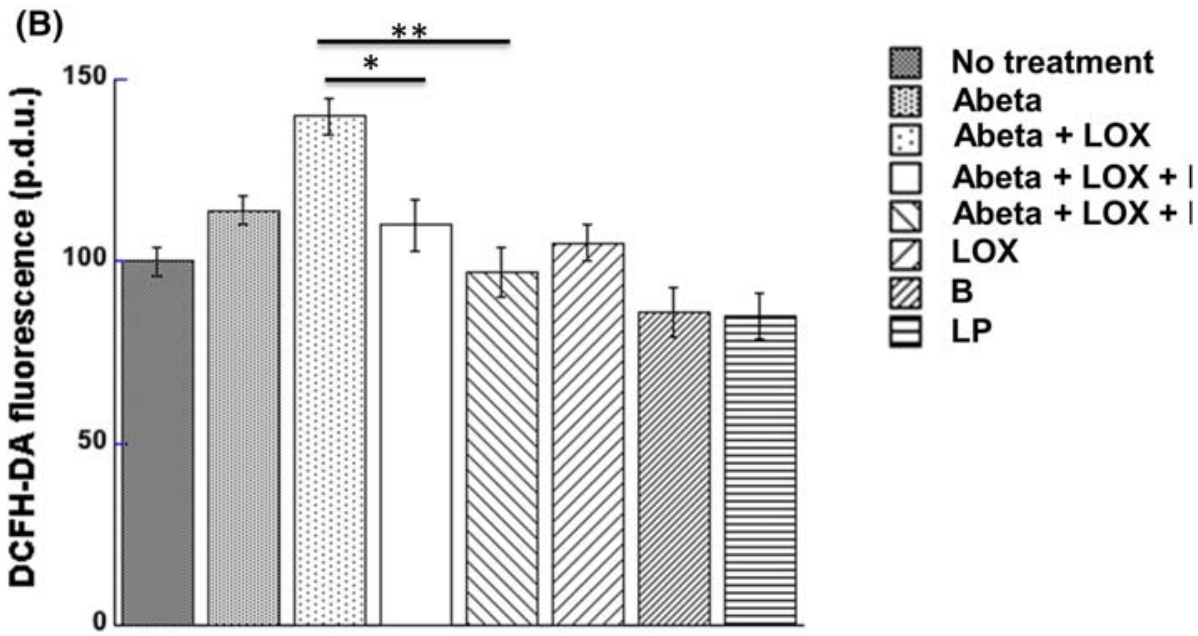

FIGURE 6 Effect of abeta induced oxidative stress on hBMVEC with and without extracellular enzymes and small molecules. (A) Oxidative stress induced by Abeta peptide $(2 \mu \mathrm{M})$ in the presence of transglutaminase $(0.005 \%)$, and osmolytes I-proline and betaine at $5 \mathrm{mM}$ cocentrations.

(B) Oxidative stress induced by Abeta peptide $(2 \mu \mathrm{M})$ in the presence of lysyl oxidase peptide $(0.005 \%)$ and osmolytes I-proline and betaine of $5 \mathrm{mM}$ concentrations. Oxidative stress is significantly reduced in the presence of osmolytes
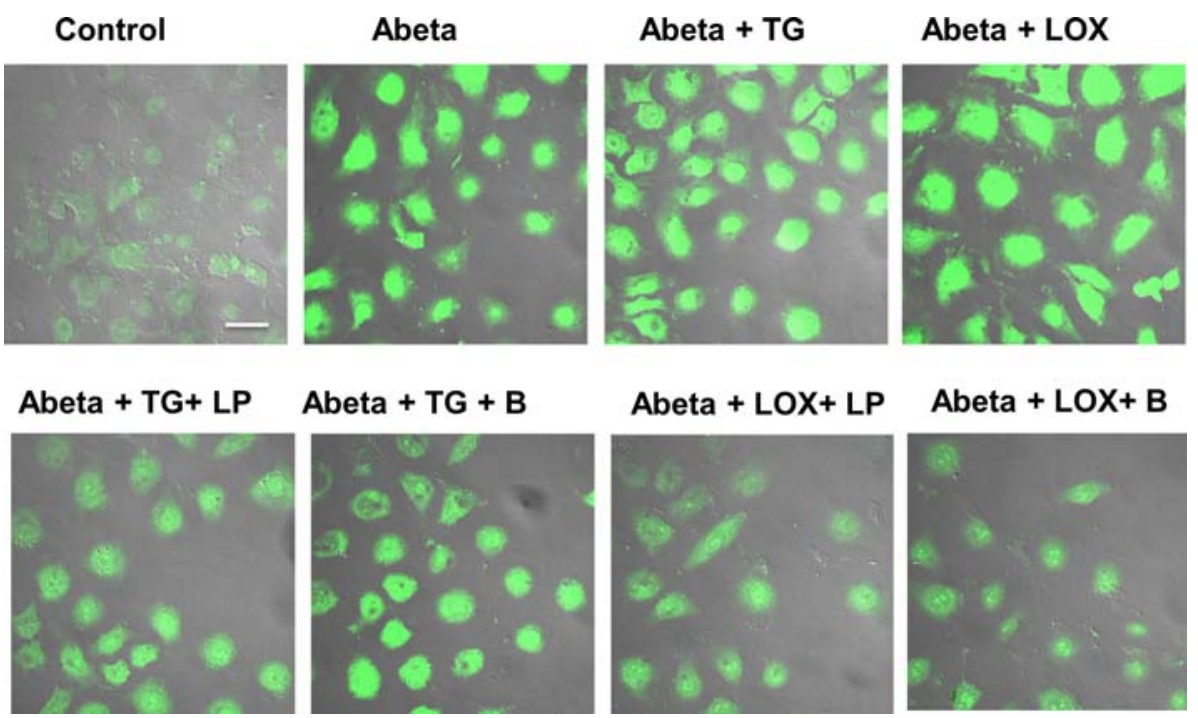

FIGURE 7 Effect of abeta-induced oxidative stress on hBMVEC with and without extracellular enzymes and small molecules, imaged by CellROX green fluorescence. Oxidative stress significantly increased in the presence of abeta, TG, and LOX, while the presence of osmolytes seems to significantly reduce the oxidative stress. Scale bar $10 \mu \mathrm{m}$ 

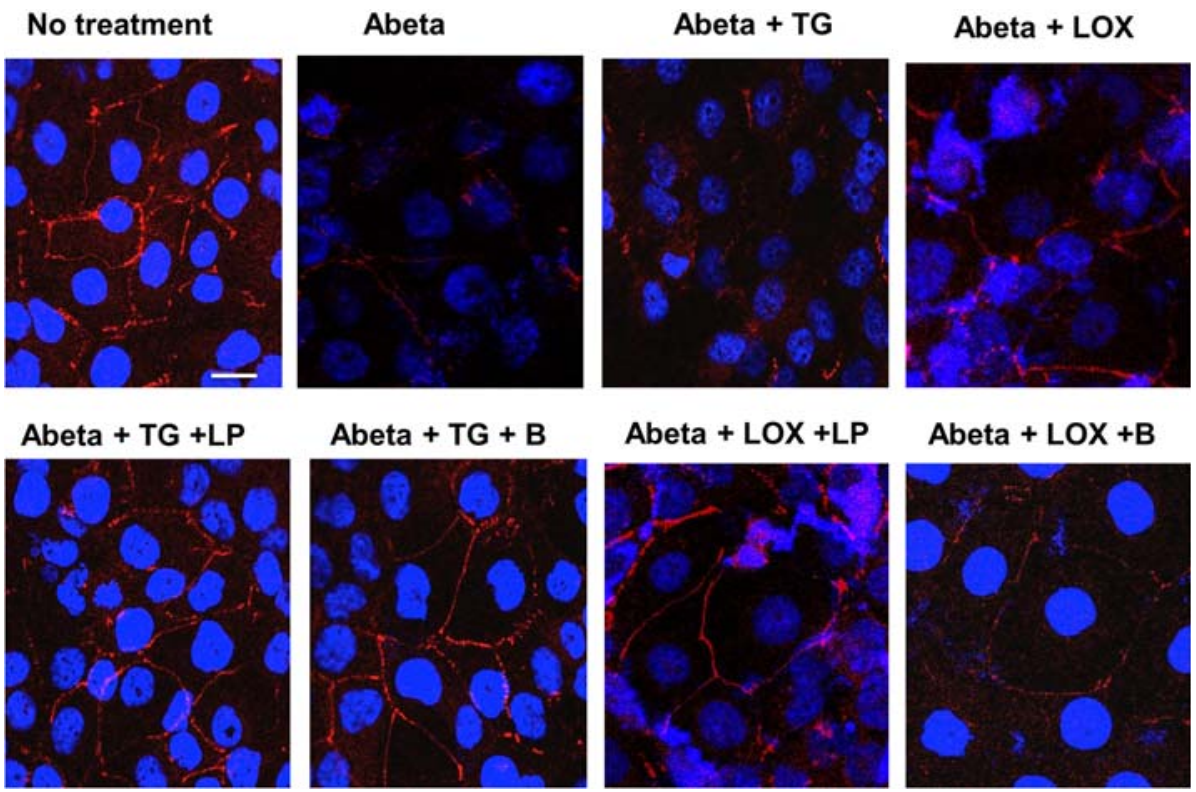

FIGURE 8 Blood brain barrier damage induced by abeta peptide $(2 \mu \mathrm{M})$ aggregation in the presence of transglutaminase and osmolytes I-proline and betaine, visualized by tight junction protein ZO-1. Tight junction is significantly restored in the presence of osmolytes. Scale bar $10 \mu \mathrm{m}$

(A)
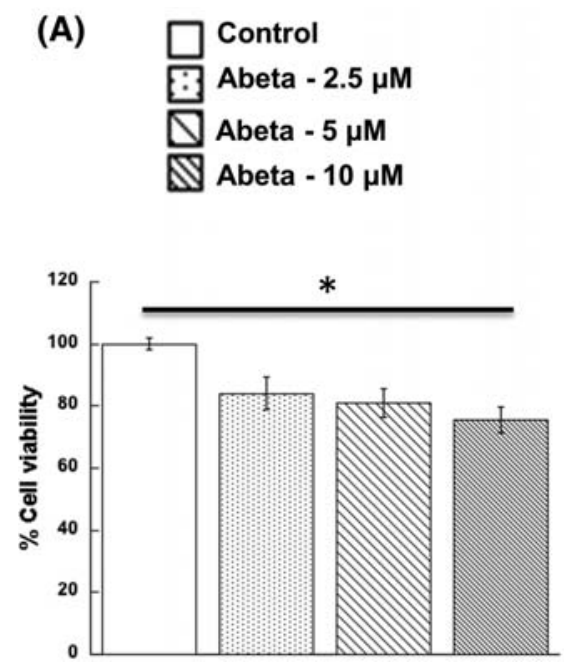

(B)

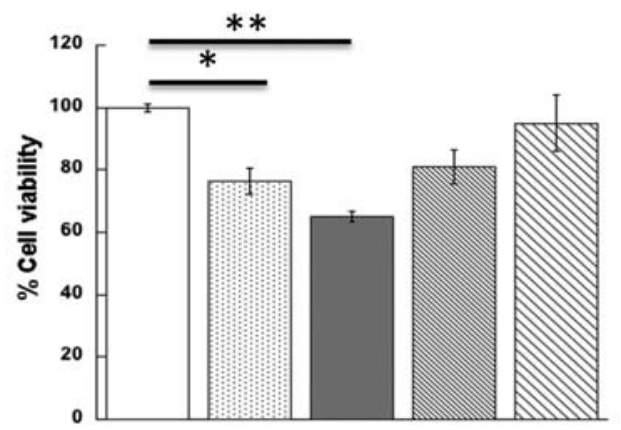

$\square$ Control

8 Abeta $-10 \mu \mathrm{M}$

Abeta + TG

$\mathbb{N}$ Abeta + TG+ LP

$\triangle$ Abeta + TG+ B
(C)
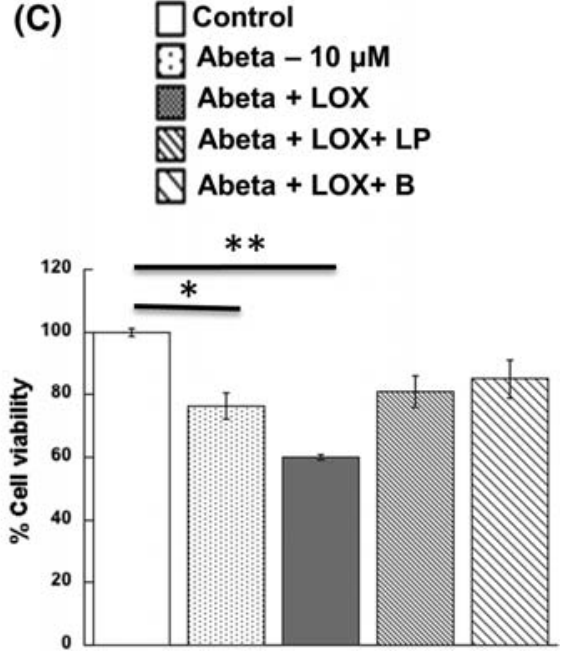

FIGURE 9 Assessment of toxicity effect of abeta peptide aggregates with and without extracellular enzymes and osmolytes in human brain microvascular endothelial cells. (A) Effect of abeta concentration, (B) effect of TG (0.02\%) and osmolytes (20 mM) on the toxicity, and (C) effect of LOX $(0.02 \%)$ and osmolytes $(20 \mathrm{mM})$ on the cell toxicity. Significant difference in toxicity is observed when abeta is treated with TG or LOX, and the toxicity effects were inhibited by I-proline and betaine

aggregation and oxidative stress of abeta peptide could be minimized. Osmolytes are known to favor compact protein conformation thereby preventing protein aggregation. ${ }^{[37]}$ Here, I-proline and betaine may have preferentially hydrated the abeta peptide, and subsequently prevented peptide-peptide interaction and aggregation. The proof of principle study reported here reveals that the osmolytes have the ability to prevent the aggregation due to extracellular enzymes. Hence, the application of osmolytes could be applied to inhibit or prevent extracellular enzymes mediated aggregation/fibrillization and oxidative stress, and potential long-term neuronal toxicity effects.

\section{ACKNOWLEDGMENT}

We would like to thank the University of Michigan Dearborn department of Mechanical engineering, and the College of Engineering and Computer Science for funding. Also, we would like to thank the University of Michigan Microscope and Imaging (MIL) facility for the use of confocal and transmission electron microscopes.

\section{CONFLICT OF INTERESTS}

The authors declare no conflicts of interest. 


\section{ORCID}

Mathumai Kanapathipillai (D) https://orcid.org/0000-0001-8748-6848

\section{REFERENCES}

[1] G. Bitan, M. D. Kirkitadze, A. Lomakin, S. S. Vollers, G. B. Benedek, D. B. Teplow, Proc. Natl. Acad. Sci. U. S. A. 2003, 100, 330.

[2] J. Drake, C. D. Link, D. A. Butterfield, Neurobiol. Aging 2003, 24, 415.

[3] M. Chen, H. L. Fernandez, Front. Biosci. 2001, 6, E1.

[4] V. J. De-Paula, M. Radanovic, B. S. Diniz, O. V. Forlenza, Subcell. Biochem. 2012, 65, 329.

[5] M. S. Wolfe, Int. Immunopharmacol. 2002, 2, 1919.

[6] S. A. Funke, D. Willbold, Curr. Pharm. Des. 2012, 18, 755.

[7] H. Li, F. Rahimi, G. Bitan, ACS Chem. Neurosci. 2016, 7, 845.

[8] J. Caceres, E. Brandan, J. Cell. Biochem. 1997, 65, 145.

[9] R. Naylor, A. F. Hill, K. J. Barnham, Eur. Biophys. J. 2008, 37, 265.

[10] R. Salza, C. Lethias, S. Ricard-Blum, J. Alzheimers Dis. 2017, 56, 991.

[11] M. M. Wilhelmus, J. G. Bol, S. G. van Duinen, B. Drukarch, Exp. Gerontol. 2013, 48, 109.

[12] D. S. Wang, D. W. Dickson, J. S. Malter, Int. J. Clin. Exp. Pathol. 2008, 1, 5.

[13] G. V. Johnson, T. M. Cox, J. P. Lockhart, M. D. Zinnerman, M. L. Miller, R. E. Powers, Brain Res. 1997, 751, 323.

[14] G. M. Gilad, H. M. Kagan, V. H. Gilad, Neurosci. Lett. 2005, 376, 210.

[15] M. de Jager, B. van der Wildt, E. Schul, J. G. Bol, S. G. van Duinen, B. Drukarch, M. M. Wilhelmus, Neurobiol. Aging 2013, 34, 1159.

[16] D. M. Hartley, C. Zhao, A. C. Speier, G. A. Woodard, S. Li, Z. Li, T. Walz, J. Biol. Chem. 2008, 283, 16790.

[17] L. G. Paleg, G. R. Stewart, J. W. Bradbeer, Plant Physiol. 1984, $75,974$.

[18] S. Choudhary, N. Kishore, R. V. Hosur, Sci. Rep. 2015, 5, 17599.

[19] M. Dasgupta, N. Kishore, PLoS One 2017, 12, e0172208.

[20] H. Wang, J. A. Joseph, Free Radic. Biol. Med. 1999, 27, 612.

[21] A. Gomes, E. Fernandes, J. L. Lima, J. Biochem. Biophys. Methods 2005, 65, 45.

[22] A. M. Swomley, S. Forster, J. T. Keeney, J. Triplett, Z. Zhang, R. Sultana, D. A. Butterfield, Biochim. Biophys. Acta 2014, 1842, 1248.
[23] D. A. Butterfield, A. M. Swomley, R. Sultana, Antioxid. Redox Signal. 2013, 19, 823.

[24] S. W. French, A. S. Mendoza, Y. Peng, Exp. Mol. Pathol. 2016, $100,426$.

[25] X. Liang, L. Zhang, S. K. Natarajan, D. F. Becker, Antioxid. Redox Signal. 2013, 19, 998.

[26] W. Wan, L. Cao, L. Liu, C. Zhang, B. Kalionis, X. Tai, Y. Li, S. Xia, J. Neurochem. 2015, 134, 382.

[27] S. Y. Kook, H. Seok Hong, M. Moon, I. Mook-Jung, Tissue Barriers 2013, 1, e23993.

[28] S. Marco, S. D. Skaper, Neurosci. Lett. 2006, 401, 219.

[29] W. F. Goure, G. A. Krafft, J. Jerecic, F. Hefti, Alzheimers Res. Ther. 2014, 6, 42.

[30] E. Hubin, N. A. van Nuland, K. Broersen, K. Pauwels, Cell. Mol. Life Sci. 2014, 71, 3507.

[31] M. C. Lampi, C. A. Reinhart-King, Sci. Transl. Med. 2018, 10, eaao0475.

[32] M. M. Wilhelmus, A. M. van Dam, B. Drukarch, Eur. J. Pharmacol. 2008, 585, 464.

[33] Z. Ignatova, L. M. Gierasch, Methods Enzymol. 2007, 428, 355.

[34] M. F. Roberts, Saline Syst. 2005, 1, 5.

[35] P. H. Yancey, J. Exp. Biol. 2005, 208, 2819.

[36] A. R. Im, Y. H. Kim, M. R. Uddin, S. Chae, H. W. Lee, Y. H. Kim, Y. S. Kim, M. Y. Lee, Cell. Mol. Neurobiol. 2013, 33, 625.

[37] F. Macchi, M. Eisenkolb, H. Kiefer, D. E. Otzen, Int. J. Mol. Sci. 2012, 13, 3801.

How to cite this article: Ismail $T$, Vancha SR, Kanapathipillai M. L-proline and betaine inhibit extracellular enzymes mediated abeta 1-42 aggregation, oxidative stress, and toxicity. Pept Sci. 2018;110:e24093. https://doi.org/10. 1002/pep2.24093 